\title{
Machiavelli e il centralismo politico intorno al viceré Luigi Lacerda y Aragón
}

\section{Silvio Suppa}

Università degli Studi di Bari «Aldo Moro»

silviosuppa@virgilio.it

\begin{abstract}
Una delle forme di manifestazione di Machiavelli nella storia e nella cultura spagnola è sicuramente l'esperienza particolarmente significativa del viceré di Spagna a Napoli, il duca di Medinacoeli. Questi operò nella città partenopea a cavallo fra la fine del 1600 e l'esordio del secolo successivo, in un momento particolarmente florido della vita civile e della produzione intellettuale napoletana, che già si era intensificata a partire dalla prima metà del seicento. L'Accademia di Medinacoeli, dal nome del nobile che la volle, si rivelò un luogo di prezioso incontro fra culture e istanze ideali differenti, tutte sostanzialmente ruotanti intorno alla possibilità di definire un modello di Stato moderno e adeguato alle dimensioni che la città aveva assunto grazie anche alla presenza spagnola. Al centro di questo raffinato circolo di professori e scienziati, non poteva mancare, o in termini adombrati, $o$ in menzione esplicita, sebbene assai problematica, il nome del Segretario fiorentino, che finì così col diventare un punto di riferimento fondamentale nella ricerca di una consapevole teoria del governo e dei compiti del governatore. Naturalmente, le tesi contenute nelle lezioni recitate a Corte — da cui il nome di Accademia Palatina o di Palazzo - si ponevano in guise differenti rispetto a Machiavelli, adottato come esempio di innovazione risolutiva, o come modello da non imitare, per la durezza che veniva imputata all'autore rinascimentale. Rimane, però, storiograficamente corretta l'impressione secondo la quale, dopo un intero secolo -quello barocco- di netta ispirazione antimachiavellica, l'occasione offerta dal Viceré di Spagna, foriera di un'apertura più coraggiosa sul Fiorentino, sembrava intervenire come un momento di inversione di tendenza e di apertura agli scritti di Machiavelli, ma soprattutto di sua possibile adozione in seno al tema dell'educazione del principe e della centralità del comando.
\end{abstract}

Parole chiave: Machiavellismo, centralismo, governo, guerra, Principe, Stato.

\section{Abstract}

One of the ways in which Machiavelli is apparent in Spanish history and culture is without doubt the especially important experience of the viceroy of Spain in Naples, the Duke of Medina Coeli. He acted in the "Parthenopean» city from the end of the XVII century to the beginning of the XVIII, at a particularly fresh time in civilian life and Neapolitan intellectual output, which had become stronger since the first half of the XVII century. The Medina Coeli Academy, named after the nobleman who requested it, proved to be a valuable meeting place for different cultures and authorities, which all revolved around the 
possibility of defining a State model that was both modern and appropriate in terms of the size the city had become with the Spanish presence as well. At the centre of this refined circle of professors and scientists, the name of the Secretary of Florence cannot be omitted, either in implicit terms or by explicit and very problematic mention. He ended up being a principle reference in the search for a conscious theory of the government or of the roles of the governor. Naturally, the theses contained in the classes recited in the Court -from which came the name Academia Palatina or Palacio - related to Machiavelli in different ways, taking him as an example of resolving innovation, or as a model not to be copied, due to the harshness of which the Renaissance author was accused. Nevertheless, remaining historiographically correct is the impression according to which, after an entire century of clear anti-Machiavellian inspiration — that of the Baroque-, the opportunity provided by the viceroy of Spain, who presaged a more courageous openness towards that of Florence, seemed to intervene as a time of trend reversal and of openness towards the writings of Machiavelli, but especially, of his possible adoption into the heart of the prince's education and the centre of command.

Key words: Machiavellianism, centralism, government, war, Prince, State.

Quando, pochi anni dopo la Congiura del Principe di Macchia (1701), il nobile napoletano, ma di nascita genovese, Paolo Mattia Doria inizia la stesura del suo manoscritto sulle «massime» del governo degli Spagnoli in Napoli, dimostra sin dalla prima pagina di aver esaminato con attenzione gli scritti di Machiavelli, soprattutto in riferimento alla figura del Principe «nuovo»e, inoltrandosi nel testo, a proposito dei metodi più indolori per mutare le leggi già esistenti da parte di un Sovrano che subentri ad altra dinastia. ${ }^{1} \mathrm{La}$ figura di un sovrano innovatore - ma se ne potrebbero citare molte altre- non a caso si profila di marcata importanza nella scrittura di Doria all'indomani dell'uscita degli Spagnoli da Napoli e aiuta non poco a comprendere quanto il «nodo» Machiavelli sia avvertito nel contesto napoletano, fino al punto da proporsi come la metafora di un'epoca che cambia, e come un modello letterario intorno al quale far ruotare tanta parte della dialettica sull'esercizio dell'arte del governo. In questo senso, e procedendo anche lungo le modalità di apprendimento dell'opera del Segretario fiorentino, le lezioni tenute presso l'Accademia di Palazzo, voluta dal Viceré spagnolo di Napoli, Luigi Lacer-

1. Lopera in questione di Paolo Mattia Doria, ha riscosso sempre un grande interesse presso gli studiosi del viceregno spagnolo dell'Italia meridionale, e in anni non più tanto recenti essa è stata resa in un'edizione a stampa corretta e filologicamente documentata: Paolo Mattia DORIA, Massime del governo spagnolo a Napoli, introduzione di Giuseppe GALASSO, testo e note a cura di Vittorio CONTI, Napoli: Guida, 1973. Il titolo esatto di questo saggio molto articolato, rimasto manoscritto sino alla pubblicazione ora menzionata, è Relazione dello stato politico, economico e civile del Regno di Napoli nel tempo chè stato governato da i Spagnuoli, prima dell'entrata dell'armi tedesche in detto Regno. Si tratta di un'opera stesa all'indomani della sostituzione della Casa d'Austria al governo di Spagna e, in particolare, sulla base di argomenti precisi che qui non è importante ricostruire, in un'epoca da collocare nel 1709. A quella data, è già in stampa la prima edizione della celebre opera dello stesso Doria La vita civile, che circolerà poi soprattutto nella veste editoriale del 1710, in un unico volume con l'altra sua importante scrittura politica L'educazio- 
da y Aragón, Duca di Medinacoeli, rappresentano tuttora un originale incrocio fra politica e cultura, unanimemente ritenuto dagli storici espressione di uno dei momenti più elevati della complessiva presenza della Spagna nel Mezzogiorno italiano. È possibile decifrare il senso di questi pochi anni, a cavallo fra XVII e XVIII secolo, quando - per una singolare e feconda congiuntura intellettuale- a Napoli è in vigore il governo di Spagna, attentissimo ai problemi politici sollevati dalle tendenze centrifughe dei signori feudali, e alla prima definizione di un'azione di contrasto a tali forze. Ë questa la temperie in cui prende consistenza una pluralità di culture e di tendenze filosofiche, ora intrecciate fra loro, quasi in una permanente contaminazione, ora sensibili alle maggiori linee di dibattito scientifico presenti in tanta parte dell'Europa delle scienze e del pensiero. Già sulla base di questi elementi molto generali, si può parlare di un preciso e intenzionale esperimento di fusione dei percorsi di conoscenza, raccolti nel tessuto intellettuale napoletano, con il campo più genuino della politica; e così emerge il segno peculiare del Duca Viceré, dettato dalla sua fiducia circa la possibilità di spingere il linguaggio della politica dentro le differenti forme della cultura moderna, nonché di mescolare quest'ultima con i significati — nemmeno tanto velati- relativi a questioni concrete di scelte e indirizzi da adottare in seno alle decisioni di corte e al controllo di una feudalità sempre pronta ad accentuare le forze centrifughe e disgregatrici della compattezza del viceregno.

Dalle multiformi esposizioni degli intellettuali invitati a tenere lezione a Palazzo reale, non è facile ricostruire immediatamente la presenza di Machiavelli e i richiami dei suoi precetti più significativi, anche perché la traccia del politico fiorentino si insinua in tante conferenze, ma il suo nome è menzionato con cautela e talvolta quasi mascherato dentro discorsi più ampi e avvolgenti. Inoltre, l'autore del Principe viene adottato, dentro un quadro sfumato di legittimazione, come "padre» di teorie di valore essenzialmente pratico, o - ancora - di scritture da interrogare esplicitamente sul piano dei concetti e dei grandi princìpi, ma alla maniera di classici antichi della politica, quasi allo scopo di evitare l'impressione di un Machiavelli immediatamente trasformabile in dottrina efficace sia per le questioni quotidiane, e sia nella sfera della teoria. Per altro, come viene fatto notare da una parte attenta degli studi sul Duca

ne del Principe. In questo denso volume del 1710 Machiavelli è menzionato ripetutamente, sebbene mediante una tecnica di perifrasi e di allusioni molto cariche di ipocrisia letteraria; ma anche la denominazione esplicita del Fiorentino ritorna in più luoghi, specialmente a proposito dell'esercizio della forza. Se si tiene conto di tale duplice saggio, di notevole fortuna negli ambienti intellettuali napoletani, e dello studio manoscritto prima citato, rimasto inedito sino al XX secolo, pressoché coevo, risulta evidente che il nome del Segretario fiorentino in Napoli è più che conosciuto, soprattutto in un arco tematico che muove dalla determinazione di cui deve dar prova il Principe nuovo al suo esordio, e giunge alla prudenza nel lavoro di legislazione successiva alla realizzazione del principato, suggerita da Doria nei modi di un mutamento lento e progressivo, e dunque quasi inavvertibile da parte dei cittadini. Questa apprezzabile circolazione di Machiavelli, trova un momento cruciale, anche se non esclusivo, proprio nell'esperienza del Duca di Medinacoeli e nel dibattito che essa riesce a liberare. 
di Medinacoeli, le diverse inclinazioni culturali da questi espressamente sollecitate non sempre corrispondono a sedimentate conoscenze in forma di sistema filosofico, o di suggerimento nel governo dell'economia; al contrario, tali conoscenze assai spesso si confondono con atteggiamenti alla moda, curiosità di ordine naturalistico, o correnti di pensiero rapsodiche, poco riconoscibili e ben celate dietro un velo di provvisorietà o di prudenza espressiva, per timore dell'ancora viva Inquisizione, o per evitare che l'autorità ecclesiastica sospetti eccessive aperture allo scientismo moderno.

Proprio il tema dello scientismo, e tutta la cospicua esposizione di un certo sperimentalismo che si rincorre in tante lezioni di corte, rappresenta infatti, e forse anche nelle più riposte intenzioni del Duca, il momento di una prima diffusione di temi eruditi, atti ad accendere un linguaggio elitario ma ben orientato al progresso o, quantomeno, alla lenta rimozione delle idee imposte dalla vecchia Scolastica. Tuttavia, esiste un equivoco di una certa importanza, a proposito della nostra Accademia, nel quale è incorsa anche una parte della più recente critica italiana, cui va però riconosciuto il notevole merito di aver pazientemente rimesso insieme tutti i testi originali delle lezioni effettivamente pronunciate. $^{2}$

Il primo orientamento da evitare, nel quale è peraltro caduta una parte della critica italiana, a sommesso avviso di chi scrive è che questo fiorire rigoglioso di pensiero civile sia la cornice in cui si affievolisce il peso di Machia-

2. Si veda la bella edizione a cura di Michele RaK, Lezioni dell'Accademia di Palazzo del duca di Medinaceli (sic) (Napoli 1698-1701), Tomi I-V, Napoli: Istituto Italiano per gli Studi Filosofici, 2000-2005 (da ora in poi Lezioni). Il gruppo di lavoro coordinato da M. Rak ha contribuito al recupero dell'intera serie dei manoscritti originali conservati presso la Biblioteca National di Madrid, e l'ha corredata di un vasto apparato di note e di eventi storici di collegamento. Copia di tutte le lezioni, raccolte da Nicola Sersale (uno dei nobili convenuti a corte, autore a sua volta di alcune conferenze), è invece conservata presso la sezione «manoscritti e rari» della Biblioteca Nazionale di Napoli. Non e fuori luogo rammentare che, nella raccolta napoletana, il frontespizio manoscritto reca la lezione latina del titolo del Viceré, riportato come Duca di Medinacoeli, in luogo di quella "Medinaceli» assunta nella redazione di Rak. Nella importante pubblicazione a cura di quest'ultimo, esiste anche un ricchissimo apparato di indicazioni bibliografiche che segnalano la serie integrale delle letture effettuate in sede storica e critico-letteraria dell'esperienza dell'Accademia, detta anche "Palatina». Nel presente contributo non è particolarmente importante ripercorrere tutto l'arco di tali scritture, per altro assai pregevoli, anche se non sempre condivisibili nella loro varietà interpretativa, fra l'idea di un'iniziativa del Viceré piuttosto rituale e imitativa di altre esperienze europee, e la tesi della leggibilità di un intento politico-civile, come vera essenza del colto circolo voluto dal Lacerda. Un primo importante livello interpretativo riguarda proprio Machiavelli, il cui nome a corte, pur talvolta velato, come già si è detto, è tuttavia presente anche in certo spirito della sua opera, sul quale il ragionamento viene ripreso con intenzione critica nel corpo - e non in nota- del percorso che qui viene proposto. Ma in via generale, è del tutto evidente che il Lacerda non rimanga indifferente alla circolazione europea delle idee e delle filosofie preilluministiche; egli quasi certamente ha tentato una certa emulazione, più che una vera e propria imitazione, degli esempi di Francia, a partire dal Re Sole, e di Inghilterra, pensando alle molte accademie di filosofi, intellettuali e pensatori di varia estrazione. Non sembra invece convincente l'impostazione di fondo del saggio di M. Rak, che fa da postfazione ai testi recitati dai tanti 
velli, particolarmente nella Napoli spagnola, a seguito di una sorta di aggiornamento delle principali domande di progresso e ordine politico dedotte dalla tradizione rinascimentale italiana, e che da essa si muoverebbero verso l'interesse per la storia e, soprattutto, per le scienze e per la natura. Nessun dubbio che il Rinascimento sia comunque in grado di supportare una ricchezza di studi e di letterature eccezionali, se si parla della figura topica della città, del governo del Principe e della vita civile complessiva; ${ }^{3}$ ma un simile riferimento appare piuttosto generico se adottato anche in questa Napoli fra la fine del Seicento e il dischiudersi della sua incredibile "avventura» filosofica settecentesca, ovvero fra il giovanissimo Giambattista Vico e il riformismo meridionale ${ }^{4}$, ancora, fra lo stesso Vico e lo storicismo critico del Cuoco. Invece, a taluni oggi è sembrato possibile ricostruire un collegamento diretto fra una nuova valorizzazione della cultura rinascimentale, e l'approfondimento della modernità nell'Italia meridionale, senza soppesare opportunamente il ruolo che, dal tardo Cinquecento e sino a tutto il secolo barocco, ha giocato la tradizione del machiavellismo e dell'antimachiavellismo, non senza trascurare l'incidenza della letteratura della ragion di Stato, anch'essa oggetto di citazioni abbastanza frequenti nelle conferenze nell'Accademia di

autori presenti nei volumi citati su Medinacoeli. Qui infatti appare predominante, più che semplicemente circolante, la tesi di un momento napoletano di grande enfasi intellettuale, dettata sia dallo sforzo di mantenere attiva un'antica tradizione letteraria della città, sia dalla sensibilità, di questi ambienti, alla storia come cultura dell'autorità, sia —infine - dalla sensibilità illuministica (o preilluministica) verso la nuova stagione delle scienze naturali che si apre, al compimento del XVII secolo, sulla stregua delle suggestioni provenienti dalla filosofia razionalistica di Francia, o dall'affermazione dell'empirismo e dello scientismo nella cultura inglese. Nessuno può negare che i modelli di conoscenza e di critica all'interno dell'intelligenza della mitteleuropa e dell'Inghilterra non abbiano esercitato un certo richiamo nella cultura napoletana - basti pensare al "renatista» Tommaso Cornelio, che generalizza in Napoli Descartes e Galilei-, ma nella città si intravedono anche altre motivazioni, nate dal bisogno autentico di fornire la forma di un sapere dedicato alla vita civile e alla costruzione di un contesto dotato di ben precise caratteristiche nella concezione dello Stato e nell'aggiornamento dei saperi più capaci di un ammodernamento delle mentalità e dei comportamenti intorno alla Corte. E in un simile ambiente, molto forte è l'esigenza di porre in ombra la cultura cosiddetta «investigante», per optare a favore di un uso decisamente politico della conoscenza. Lo sperimentalismo e la qualità fisica del concetto di natura interpretato dalla corrente detta degli "Investiganti» (dal nome dell'Accademia degli Investiganti, nata nel 1650 e terminata nel 1656 a causa della peste), alla fine del secolo appare un corpo di idee inclini più alla frammentazione, se non ad una sorta di occasionalismo scientifico, che alla produzione di concetti e di culture funzionali a ricomporre la centralità dello Stato.

3. A questo proposito, su tutte, valgano le fondamentali indicazioni di Eugenio GARIN nelle seguenti opere: L'Umanesimo italiano. Filosofia e vita civile nel Rinascimento, Bari: Laterza, 1952; La cultura filosofica del Rinascimento italiano, Bari: Laterza, 1961; Scienza e vita civile nel Rinascimento italiano, Bari, Laterza, 1965; La cultura del Rinascimento, Bari: Laterza, 1967, ed altre ancora. Si veda ancora di Ernst CASSIRER, Individuo e cosmo nella filosofia del Rinascimento, Firenze: La Nuova Italia, 1927.

4. Rinvio, a questo proposito, alla vivace opera di Nunzia Di MASO, Il repubblicanesimo di Vincenzo Cuoco. A partire da Machiavelli, Firenze: Centro Editoriale Toscano, 2005. 
Palazzo. ${ }^{5}$ Siamo in anni in cui negli ambienti intellettuali di Napoli è più consigliabile occultare, o almeno porre nell'ombra, il nome di un filosofo come Spinoza, per fare un esempio, pensatore molto discusso e persino tacciato di sostanziale ateismo, che entrare nel merito della differenza fra il dualismo delle «sostanze» cartesiane (res cogitans e res extensa) e la veduta unitaria del discusso pensatore olandese. Allo stesso modo, in questo ambiente sembra che sia meglio muovere da un tono cauto nel recupero del senso forte del Segretario fiorentino, mentre la sua circolazione si avvia già ad una crescita progressiva e destinata a spingersi sino a tutto il XVIII secolo nei modi di un simbolo culturale molto citato e molto rivisitato. Tenuto conto di un simile clima di tensione attiva fra la prudenza nei confronti della tradizione, e la spinta intellettuale verso una più esplicita adozione degli auctores della politica, la pagina del Lacerda merita una più attenta considerazione, soprattutto per il fatto che la grande sensibilità —anche un po' opportunistica- del Duca, verso la cultura civile dei nuovi ceti illuminati, interviene come un'occasione non ripetibile di dialogo fra passato e presente, e per altro completamente liberata da ogni soggezione a differenti tendenze di pensiero assai più incerte sul nodo della concezione e della funzione centralistica dello Stato; si pensi, per fornire un nome di indubbio spessore nella parte più avanzata del ceto forense napoletano, a Francesco d'Andrea e al suo patrimonio di giusnaturalismo, che nel cuore del Seicento aveva contribuito a diffondere un diritto delle garanzie, piuttosto che una costruzione giuridica dell'ordine. ${ }^{6}$ Alla fine del secolo, la virata del pensiero intorno alle istituzioni e alla funzione del Principe appare un ritorno quasi «ufficiale» all'evidenza della politica, che riesce a definire la sua ingombrante attualità anche quando i suoi elementi più pregnanti si esprimono attraverso gli abiti della storia, o dell'arte militare, o della metafisica, come avviene, per quest'ultimo genere speculativo, nella prolissa opera di Doria.

La questione del centralismo politico, su cui si incentra il presente contributo, è in effetti il "fuoco» critico intorno al quale si accende il circolo acca-

5. La tesi del superamento di Machiavelli, anzi della sua «fine», apre un paragrafo del saggio di Rak interno al volume Lezioni, cit.; si veda: Michele RAK, L'Accademia di Palazzo del duca di Medinaceli. Un'accademia di storia, antiquaria, scienza e letteratura a Napoli (16981701). Questo studio apre il Tomo V della più volte menzionata edizione a cura dello stesso Rak, dove a p. 74 prende forma la tesi - poco convincente in verità- circa la percezione in Napoli di Machiavelli come un nome «che non era un teorico pericoloso, era un teorico d'altri tempi» (op. cit., p. 75). La medesima tesi torna ancora nel paragrafo intitolato «Senza Machiavelli» (ibidem, p. 104), dove il sottile rifiuto del "perversi» consiglieri del Principe, esercitato in una dotta lezione di Nicola Sersale, è stato interpretato in termini di una distanza dal Fiorentino, messo da parte, se non escluso, dall'analisi della storia, che oramai domanderebbe una nuova impostazione intellettuale e inaugurerebbe una diversa stagione della cultura e della politica.

6. Su Francesco D'Andrea si veda Biagio De GIovanni, Filosofia e diritto in Francesco D'Andrea. Contributo alla storia del previchismo, Milano: Giuffré, 1958; Salvo MaSTELLONE, Francesco D'Andrea politico e giurista (1648-1698). L'ascesa del ceto civile, Firenze: Olschki, 1969. 
demico promosso dal Viceré; ma intorno al medesimo argomento insorgono dei filtri che non aiutano a rivelare le diverse metafore del potere prodotte in questo ambiente, dove i linguaggi sono estremamente differenziati e sono particolarmente articolati in una gamma di contenuti che la dialettica fra natura e storia non riesce ad esprimere interamente. Innanzitutto va considerato che l'Accademia viene istituzionalizzata dallo stesso Duca, e da lui convocata due volte al mese, per dare un segno di congiunzione fra il ruolo istituzionale del Principe e la sua effettiva intenzione di divenire il promotore dell'arricchimento della conoscenza, se non del governo dei filosofi, sia che questi ultimi si mostrino attenti ai moderni, sia che si propongano eredi e interpreti fedeli, ma non speculari, dei tempi antichi. Tuttavia, non va dimenticato che nella Napoli fra Sei e Settecento la polemica intorno ai moderni è serratissima e tutt'altro che univoca; pertanto, in un simile rigoglio di temi e di filosofie, naturalistiche e civili, il problema dell'ammissione a corte del ceto intellettuale, nelle intenzioni del Duca di Medinacoeli, nasce dal suo stesso disegno di mantenersi alleata la parte più avveduta della nobiltà e della borghesia intellettuale di Napoli, così come di proporsi ad autentico cultore degli studi, e di creare un incontro a mezza via fra la centralità del sovrano, e la mentalità di consenso fornita dalla parte più raffinata dei sudditi influenti e dei maggiori esponenti delle alte professioni e delle accademie. Su tale assunto di fusione fra politica e saperi si spiega, nelle conferenze di Corte, la fantasiosa convivenza della storia, della filosofia morale, della letteratura e dello studio della natura, ora riflesso di un tardo giusnaturalismo di ispirazione groziana, ora segno del progresso mosso da Galileo e Bacone, e spinto sino a Newton e a quel Descartes amato e contestato al tempo stesso. Ma una simile disordinata varietà è comunque attraversata da un filo di Arianna di sapore civile e politico; perciò, la filosofia del sensismo incontra importanti remore da parte di chi, come il cartesiano Paolo Mattia Doria, molto amico di Giambattista Vico e molto in sintonia con il governo di Spagna, in essa riscontra un titolo di legittimazione per l'egoismo degli interessi particolaristici, e quindi per le forze feudali e anticentralistiche. Già questa semplice contraddizione, che è oggetto di una storiografia pluridecennale, ci riporta comunque nel vivo della questione del centralismo politico; ma si tratta solo di uno dei segni di tale questione, per altro ben mimetizzato dentro l'antico costume alla disputa, tipico del ceto intellettuale napoletano, e che meglio si dispiega nelle lezioni di carattere storico e nella loro ampiezza fra ripresa dei costumi - le «sontuose cene de' Romani» di $\mathrm{Vico}^{7}$ - e paradigma del potere forte e centralizzato nell'imperatore che governa, da solo e con abilità, territori immensi.

Esistono, dunque, diversi profili di rilievo in questo fortunato momento del viceregno spagnolo, i quali conducono sempre al problema del centralismo politico, e che si servono di precisi generi letterari, in cui si conserva un'opzione politica a favore del peso determinante del Principe nelle sorti del governo; 
e qui il patrimonio delle letture e della diffusione continentale del grande Fiorentino non si limita certo né ad un dotto ricordo, né — tanto meno- ad una citazione di astratto accademismo. La politica appare nei molti autori che riprendono il tema delle "vite dei Cesari» o, risalendo nel tempo, degli antichi imperi e di tutti i modelli in grado di riaprire il tema delle virtù del Principe, o dell'accezione eroica di questo ruolo di fondazione delle civiltà, prima ancora che di conservazione. Il Principe è il reggitore, fulcro decisivo della durata dell'impero, non senza indurre il concetto della dipendenza dell'equilibrio dalla centralità del potere e dalla sua forza senza incertezza. Le lezioni intorno al Lacerda insistono molto, forse anche troppo, sul modello imperiale, probabilmente anche con intenzione retorica verso la stessa storia della Spagna; ma il loro motivo più costante è il centralismo e l'unificazione del comando, contro le tendenze centrifughe e contro le forze che, rivendicando autonomia, portano, e hanno in effetti portato, a disgregazione la continuità del comando e quindi l'Impero tutto. Ed ecco ricostruita la questione del centralismo non come mezzo organizzativo, ma come risorsa politica per la durata e l'autorità del Principe-Imperatore.

Questa tendenza ha differenti, e spesso contrastanti, espressioni alla Corte del Duca di Medinacoeli. Troviamo il cartesiano Gregorio Caloprese - uomo di rilievo a Napoli- che respinge duramente il patrimonio machiavelliano, ridotto a trionfo casuale della "fortuna", e per giunta mescolato con tracce del naturalismo bestiale di cui parla Hobbes. Il discorso sull'origine degli imperi, di Caloprese, si radica in un modello morale, a metà fra l'ortodossia cattolica, per frenare le passioni, e la spinta soggettiva al rigetto delle ambizioni individuali. Nasce così la definizione di un sovrano ideale, prudente nei costumi e assistito da ufficiali capaci, sulla base di un esempio desunto dalla monarchia di Spagna. Afferma Caloprese: «Il nostro sempre invitto e glorioso imperatore Carlo Quinto, come havrebbe potuto conseguire tante e sì gran vittorie in Italia, in Germania, in Africa et in Ungaria, se egli non fusse stato circondato da una gran schiera di famosi capitani, chiari non meno per lealtà che per valore?». ${ }^{8}$ Il problema della forza, mista alla virtù, del gruppo fedele al «capo», è direttamente collegato alla qualità individuale del grande sovrano, vero punto di equilibrio di un Impero altrimenti impossibile. Questo percorso narrativo, di marcato centralismo moralistico, si colloca nella continuità di un antimachiavellismo secentesco, tanto più trasparente, quanto più accentuato dallo scrupolo con cui Caloprese fonde disordinatamente la condizione di natura di Hobbes con l'astuzia di Machiavelli, e si astiene con scrupolo dal citare tanto il filosofo inglese, quanto il Segretario italiano: «Niun ha mai creduto - egli scrive quasi all'inizio della sua terza lezione- che noi siamo stati creati dalla natura in guisa che noi non possiamo far altro che male»; ${ }^{9}$ e tutto il suo ragionamento è quasi una parafrasi parallela dei due autori moderni ora menzionati, ai quali contrappone il nesso antico fra politica e virtù. La dissertazione su Tiberio, tenuta da Filippo Anastasio, influente

9. Ibidem, p. 25. 
figura di ecclesiastico, è un altro esempio di antimachiavellismo — questa volta assai duro- dato che Machiavelli menzionato, se non proprio con il suo nome, almeno con l'eterno appellativo di «Segretario fiorentino», ${ }^{10}$ è pareggiato a Tiberio, ed è collocato fra i maestri di tirannia, partendo da una minuta ripresa, quasi testuale, del celebre dubbio se il Principe debba essere amato oppure temuto; la conclusione dell'Anastasio è di tipico impianto da logica dottrinaria e astratta: "Or se l'amore e 'l timore posson congiungersi — egli dice- e, travalicando da una parte, non si cade di necessità nell'altra, a che proposito tal questione proponesi e come peggio, decidesi?». ${ }^{11} \mathrm{Ma}$ i riferimenti a Machiavelli sono assai frequenti, sullo schema della dissertatio adversus Machiavellum che -com'è notorappresenta un tópos della letteratura dell'antimachiavellismo secentesco. Tuttavia, anche questo schema di contestazione minuta e dettagliata del Fiorentino, è il segno più evidente dell'attenta lettura che egli ormai riscuote anche negli ambienti che lo respingono.

Diverso è il tono quando si parla più diffusamente dell'Impero romano, che nelle lezioni rientra nella logica ciceroniana della historia magistra vitae, ma che contiene due interessanti motivi politici. Il primo si incontra nella conferenza sulla «vita di Aurelio Antonino Bassiano, detto Caracalla» tenuta dal Doria. Qui la persona dell'Imperatore viene caricata di pesante responsabilità, per aver praticato una politica senza principi morali, esageratamente prepotente, e che quindi ha causato l'indebolimento e poi la fine dell'impero. La ricostruzione di Doria sembra volere concentrare le ragioni della caduta di Roma sul ruolo decisivo e quasi personale del Cesare, per sostenere, abbastanza intenzionalmente, che la grandezza o la miseria dei più vasti sistemi di potere dipende da chi li guida, esattamente come succede anche per qualsiasi altro piccolo regno. Ora Doria introduce la questione della qualità del Principe, specialmente nel compito, che questa figura ricopre, di centro di coesione dell'impero, e apre una pagina di teoria del Sovrano in uno Stato governato contro le forze centrifughe, e cioè — nel contesto napoletano- contro i baroni proprietari e i signori dei feudi. È una teoria che il nobile genovese alimenterà per diversi anni, e che trova già nel Lacerda l'esempio concreto di una possibile concentrazione, nella Corona, del filosofo e del capitano, come Doria dirà più tardi nei suoi libri, intorno all'educazione di un di Principe dal profilo un po' platonico-ficiniano, per la filosofia, e un po' machiavelliano, per la forza. Insomma, questo intellettuale sembra avere fiducia nel Duca spagnolo al quale si rivolge anche direttamente nel corso delle sue conferenze, e sembra nutrire un'apprezzabile speranza circa la possibilità di realizzare l'avvento di un Principe Nuovo, come dice Machiavelli, e anche intellettuale, come lo stesso Lacerda intende apparire. Già l'inizio della lezione suona come una diagnosi storica, che fa dipendere la caduta di Roma «dalla licenza de' popoli e dalla sfrenatezza de' prencipi». ${ }^{12}$ Se dunque il Principe è debole, le popolazioni a lui sottomesse 
perdono il rispetto per la loro guida centrale, e coltivano interessati disegni di disgregazione civile. Se, da un lato, prende corpo la ripresa della radice più profonda dei testi di Machiavelli, insieme con la necessità di una formazione ideale e filosofica dell'uomo di governo, dall'altro lato interviene anche l'esigenza di motivare con maggiore precisione il ricorso alle armi e alla forza: affiora qui un primo riferimento inequivocabile alle regole dell'arte della guerra. È uno dei temi più importanti dell'opera di Machiavelli, in questa lezione accennato e introdotto per simboli fugaci, ma ormai decisamente affiancato al più ampio discorso sul Principe-Imperatore. Caracalla errò, infatti, perché "ostentò nella guerra — afferma Doria - tutta la sua barbarie con deboli, fece comparire tutta la sua debolezza con forti». ${ }^{13} \mathrm{Nel}$ genere storico è più facile, rispetto alle scienze naturali, introdurre argomenti di politica; di conseguenza proprio in questo versante degli interventi in Accademia filtrano interpretazioni diverse di Machiavelli, che confermano la diffusione delle sue opere, e moltiplicano il ventaglio delle posizioni intorno ai termini attuali e immediati del tema della centralità e delle virtù del Principe. Parliamo di un Machiavelli nominato attraverso i contenuti delle sue opere, e che interviene per allusioni di senso anche opposte fra loro; nella sua lezione sulla «Repubblica dell'Ebrei», per fare un riferimento significativo, Nicolò Caravita sottolinea le virtù civili di questo popolo e, a conferma, crea un'immagine di contrasto rinviando al Segretario fiorentino, definito puramente e semplicemente «il più empio politico». ${ }^{14} \mathrm{Si}$ tratta di un'espressione quasi rituale in Italia, dietro la quale si nascondono sia gli antimachiavellisti autentici, sia quelli simulati, che con la formale condanna dell'autore di Firenze, nemmeno nominabile, ne rimettono in circolo la teoria sostanziale. Questa mimetizzazione della parola era imposta a Napoli, e anche altrove, dal timore dell'Inquisizione, che tuttavia si attenuava parecchio quando, come alla corte del viceré di Spagna, si creava un clima di tolleranza e si parlava con una autocensura blanda e da mera recitazione. In una più estesa serie di lezioni sull'Impero romano, Nicola Sersale, che era anche segretario dell'accademia e ne custodiva i testi scritti, si diffonde in ripetuti passaggi su Machiavelli, espliciti oppure per il tramite di Tito Livio. Romolo, figura del fondatore e simbolo di ogni centralizzazione della politica, "Costituì [...] in Roma la religione, — dice Sersale_- dandogli più ampia e speciosa apparenza et facendo a coloro credere che le leggi da lui ordinate dalla ninfa Egeria dettate gli fussero». ${ }^{15} \mathrm{Ma}$ l'intera epoca della monarchia romana, con Numa Pompilio ripropone in modo ancora più istituzionale il culto e la funzione civile della religione, utilizzata nella sua qualità di sistema di regole e di ordine: «Stabilita dunque da Numa — afferma ancora il Sersale - et posta in sommo preggio la religione, s'indusse in Roma la pace et la quiete ne' sudditi [...]». ${ }^{16}$ Il riferimento ad alcune parti salienti dei Discorsi di Machiavelli è più che evi- 
dente, in un impianto che trasforma in massima di governo l'interpretazione della storia, dopo che anche questo genere letterario ha rivelato tutta la sua traducibilità in insegnamento politico, proprio come accade nel testo del Segretario fiorentino. Ecco perché, in questa stessa lezione, viene richiamato il celebre binomio machiavelliano dell'ordine e delle armi: $«[\ldots]$ in quelle repubbliche —afferma il Sersale parlando indifferentemente di «repubblica» anche per riferirsi alla "monarchia» — dove è religione, facilmente possono essere l'armi introdotte et ivi forza acquistare, ma per lo contrario, non mai o con grandissima difficoltà s'introdurranno non essendovi religione, benché l'armi vi siano». ${ }^{17}$ Eppure, in questa lezione non si fa certo professione di concezione machiavelliana della politica, essendo il Sersale altra cosa che un intenzionale divulgatore dell'opera del Fiorentino. Ma tale opera, sembra ormai essere diventata, se non un paradigma inconfondibile, almeno un luogo di mediazione e un percorso comune per indirizzi talvolta anche irriducibili l'uno all'altro. In effetti, con l'Accademia Palatina siamo di fronte ad un'occasione dotta, come dotto è tutto il linguaggio di queste lezioni, il quale tuttavia diventa particolarmente esplicito quando si parla di contenuti politici, proprio perché tutta la vita di un simile circolo di intelligenza civile è una non ripetibile occasione, per il Duca, di proporsi nella sua veste intellettuale migliore, e per i suoi interlocutori, di rivolgere la parola ad un vice-sovrano, disposto ad apprendere; in questo senso, tutte le lezioni a Palazzo costituiscono il robusto tessuto di una paideia del Principe che è il vero punto di approdo di tanti ragionamenti sulla conclamata centralità del massimo responsabile della vita del popolo e dello Stato. Nella duplice funzione del Duca di Medinacoeli, ora magister, e ora apprendista dei modi di governo, si agita tanta parte del dibattito sulla rilevanza primaria e fondamentale del Principe che, in questo confronto a più voci, si delinea sempre più come il problema sia dell'accentramento dei poteri di governo, contro le riottose periferie, sia dell'importanza della formazione del Principe, ancora vitale nel passaggio dal XVII al XVIII secolo. Nella medesima conferenza del Sersale, infatti, prosegue la lezione di Machiavelli, anche sul piano di più dettagliate tecniche della politica. La fase consolare di Roma, è quella in cui si perfeziona l'accesso alla vita pubblica di tutti i ceti della città, sino alla plebe compresa, che però interviene come un problema e un soggetto di inquietudine, di rischio per gli equilibri, sino alla nascita delle nuove magistrature del popolo: «Ottenne col tempo però — seguita il Sersale_ la romana repubblica la [...] perfezione se non dall'ordinatori almeno dal caso", quando per la «disunione» della plebe dal Senato, i nobili e lo stesso Senato furono costretti a dare vita ai «tribuni della plebe». ${ }^{18} \mathrm{E}$ qui anche il lessico — si parla di «disunione»— oltre che l'intero racconto storico, rivelano in modo inequivocabile un debito verso Machiavelli, in un momento politico-intellettuale in cui il problema della formazione-educazione del principe ha preso tutto il suo spazio. E infatti, lo stesso Sersale, quasi anticipando la celebre opera di Montesquieu, si interroga sulle 
cause della caduta dell'Impero Romano, e chiude la sua lunga dissertazione affermando ancora la grave responsabilità dei regnanti, nell'andamento degli imperi. La sintesi finale del ragionamento di questo accademico si esprime con un linguaggio assolutamente filtrato attraverso le pagine di Machiavelli; per Sersale, infatti, la fine di Roma lascia "un saggio documento a' regnanti che, - egli scrive - sollevati dal valore e dalla fortuna su grado eminente dell'humana potenza, stanno prossimi a' precipizii se al pari che cresce il loro dominio non si aumenta ancora del governo la cura e l'attenzione». ${ }^{19}$

Ancora i contenuti machiavelliani tornano in alcuni passaggi di Doria, nella sua conferenza sulla vita dell'Imperatore Claudio, che si apre con un preciso riferimento al ruolo e all'attitudine del sovrano. ${ }^{20}$ In questa dissertazione il termine «disunione» torna, ma nel linguaggio del Genovese sta a significare la premessa delle "lotte civili», e quindi agisce come causa di frammentazione, o di perdita della forza del Cesare, centro dell'Impero. Qui la dimensione del comando pone il necessario corollario del legame fra soldati, popolazioni e Principe, e già si affaccia un discorso ancora per simboli storici intorno alla forma più moderna e compiuta dello Stato. ${ }^{21}$ Molto più deciso è il contributo di un nucleo di altre quattro lezioni del medesimo Doria, tutte di marcata impostazione machiavelliana, e costruite come una rigorosa dottrina del ruolo "centralissimo» - direi- e delle caratteristiche anche individuali del Principe, dal punto di vista delle sue virtù e della sua stessa capacità di intervento. Ora l'arte militare - titolo di una conferenza che fa eco all'opera del Fiorentino - riconduce tutta la storia degli uomini ad una successione di relazioni polemologiche, o relazioni conflittuali, e persino il trionfo della giustizia, o il rispetto dei confini di un Paese, non possono essere assicurati senza disporre di un'adeguata capacità militare. ${ }^{22}$ In questa capacità, è compresa anche la perizia dei soldati, sia nell'uso della "violenza», sia nel suo dosaggio con la "chiarezza della mente». Questa specifica intelligenza sembra attentamene ritagliata sulla misura di un Principe nemmeno tanto immaginario, ed anzi collocato nel vivo di tensioni e di insidie all'interno, prima ancora che disposte lungo i confini con lo straniero. A tale proposito, l'arte militare di Doria si precisa anche nel senso di arma politica, in quanto parte integrante dell'azione di contrasto alle «interne discordie». ${ }^{23} \mathrm{Ma}$ la tempestività e la conoscenza delle caratteristiche proprie «delle nazioni che ha da governare» è qualità indispensabile del governatore, specialmente quando egli intenda conservare sia il suo potere, sia la sua centralità.

19. Ibidem, p. 355 .

20. Ibidem, p. 529.

21. Ibidem, p. 533.

22. Il testo di Doria, talvolta anche ripetitivo nell'incedere delle lezioni, recita: «Ella altro non è quest'arte che un violento e forte rimedio della malizia delle nostre inclinazioni, non ad altro diretta la mostruosa apparenza di quelle straggi, che avvalorando le leggi disarmate et imbelli e sforzando a vivere dentro i limiti dell'onesto la rapacità de' vicini, fare signoreggiare nel mondo l'equità, la giustizia, l'amore e tutte quante le più belle virtudi [...]». Lezioni, cit., T. III, p. 305. Il medesimo concetto ritorna ancora nelle pagine successive.

23. Ibidem, p. 312. 
Insomma, la centralità del Principe non è neanche proponibile fuori dall'aggiornamento delle qualità e della efficacia della sua forza. ${ }^{24}$

Difficile dubitare, ormai, che Machiavelli sia largamente circolante, e anche assimilato, in questo ambiente che sottopone al proprio Duca il discorso più generale del livello raggiunto dal conflitto politico, al punto da poter attribuire all'intero senso della politica moderna il valore di un'ambivalenza fra durata e fine, da sciogliere solo con la responsabilità diretta del Principe. In una certa misura Doria può essere interpretato anche come l'autore che prova a riscrivere lo statuto dell'eroe, e cioè a proporre nello stesso Principe non solo la forza del fondatore, ma anche quella della guida nella vita successiva del regno, ben consapevole del peso e del grado di impegno personale che l'esercizio dello Stato impone. La "piazza», nel senso militare, si mantiene con la forza, ovviamente, ma anche con l'armonia e con la mente filosofica. Ecco la nuova frontiera domandata al sovrano, il quale deve servirsi non più tanto della storia e delle imprese, quanto di una sua individuale inclinazione alla virtù e all'intelligenza teorica, cioè alla morale e all'esercizio della ragione insieme platonica e cartesiana. Il governatore deve perciò essere dotato di «morali virtudi, con le quali si deve rendere amato e venerato da' suoi et ammirato da' nemici». ${ }^{25}$ Le successive lezioni sul governatore di piazza e sulla scherma non modificano questo piano del discorso, se non per ulteriori precisazioni di ordine tecnico-militare, per l'eccellente equilibrio fra mente e corpo, e per l'esercizio della prefigurazione dei movimenti dell'avversario.

Non si conclude qui, ma circola anche in altre lezioni di vari autori la riscoperta di Machiavelli alla corte del Viceré di Spagna. Dopo, verrà la congiura di Macchia, e il richiamo a Madrid del Lacerda. Laccademia durerà un attimo ancora, prima che giungano gli Austriaci (1707), ma il nuovo ruolo di un'intelligenza civile costruttiva, intorno al suo sovrano e per lo Stato forte e coeso, è stato inaugurato, con il binomio di prudenza, e Machiavelli. 